\title{
A Call Level Adaptive Bandwidth Allocation Scheme Based on Lyapunov Control Theory
}

\author{
Hesham Bin-Abbas \\ Department of Electrical Engineering \\ University of Pittsburgh \\ Pittsburgh, PA 15260 \\ Email: binabbas@kacst.edu.sa
}

\author{
David Tipper \\ Telecommunications Program \\ University of Pittsburgh \\ Pittsburgh, PA 15260 \\ Email: tipper@tele.pitt.edu
}

\begin{abstract}
In this paper, we propose an adaptive capacity allocation scheme at the call level for a blocked call cleared loss system. The scheme aims to maintain the connection blocking probability around a specified target value by dynamically adjusting the allocated capacity. Based on a fluid flow model of the loss system, Lyapunov Stability theory is used to derive an adaptive capacity adjustment scheme which guarantees overall system stability around the target call blocking probability. Numerical results are given which show that the Lyapunov control based scheme is robust to load variations and performs better than existing schemes in the literature.
\end{abstract}

Keywords : Adaptive Capacity Allocation, Call Blocking, Control Theory, Fluid-Flow Models

\section{INTRODUCTION}

In this paper, we propose a novel adaptive bandwidth allocation scheme at the call level for a blocked call cleared loss system. The goal of implementing a bandwidth adjustment scheme is to provide a mechanism which will allow for efficient use of network resource while meeting QoS requirements. The basic idea is to dynamically allocate and deallocate the capacity from the underlying transport network for different calls/flows/connections/service classes in order to respond to the network traffic changes so as to meet the QoS requirements. In other words, if the traffic is low for a particular class at certain time, as long as the QoS is met, some capacity may be deallocated for use by either other services or assigned to a shared pool. Similarly, if the QoS of a certain traffic class is degraded by any network changes, additional capacity may be requested from the transport network. As a result, better utilization of the network resources can be achieved. The capabilitiy of dynamically allocating/deallocating bandwidth is now possible in some network technology (e.g., SONET Mesh Networks [4], cellular networks with dynamic channel allocation ) and is expected to be common place in the future.

Over the years a fair amount of literature has appeared on developing adaptive bandwidth control algorithms to meet packet level QoS metrics and the interested reader is referred to the survey paper [16]. There has been much less work in the literature that addresses call blocking and capacity adjustment at the call level. Mase and Imase [18] proposed adaptive bandwidth allocation for virtual trunks in circuit switched networks to handle variation in multi-hour loading patterns. A simple adjustment heuristic was proposed. Shioda [15] proposed adaptively adjusting the capacity allocated to virtual paths in ATM networks as a function of the call blocking deviation from a desired call blocking rate. A simple threshold approach on the deviations and capacity adjustment was proposed. In [7], a dynamically, reconfigurable, capacity environment was addressed. Adaptive capacity schemes based on the blocking and utilization as means to calculate when and how much adjustment should be made were proposed and a comparative performance evaluation presented. However, the assumption of a priori knowledge of the traffic, for which the adjustment parameters can be properly tuned, make the schemes not very robust.

In this paper, we adopt the same scenario of [7] and propose a robust, adaptive scheme that automatically adjusts the link capacity using a control theory approach. In past there has been interest in applying techniques from control theory to regulate communication network behavior. This work has focused on a variety of problems from IP packet level congestion control [10], [14], to ATM cell level network congestion control [3], to IP packet level bandwidth allocation [17]. Here we focus on the call or connection level network behavior. Specifically, in this paper, we present a differential equationbased fluid-flow model that captures the traffic dynamics of a loss system. Based on the fluid-flow model, Lyapunov stability theory is used to determine an adaptive bandwidth allocation scheme that guarantees an overall system stability by forcing the call blocking of the system to track a desired value or desired time varying trajectory. The resulting scheme is simple to implement and is shown to have performance which is robust to variations in the input load, parameter settings and desired trajectory.

The remainder of the paper is organized as follows. In the next section, we present a fluid-flow based model of the call blocking behavior in a loss system. In Section III, we introduce the Lyapunov Stability Theorem and its application to obtain an adaptive capacity allocation scheme. Then, in Section IV we evaluate the performance of the proposed scheme through simulations. Finally, the paper concludes in Section V. 


\section{A Fluid-Flow MOdel fOR LOSS Systems}

In order to develop an adaptive bandwidth allocation scheme based on control theory principles a suitable model of the dynamic behavior must be determined. Here, we adopt an approximate fluid-flow modeling approach described in [19] and [20]. We consider a nonstationary offered load to a single traffic class loss system. In modeling the loss system, we consider the case where the link capacity $C(t)$ is time varying and is counted as a multiple of a basic bandwidth unit (e.g., 64 Kbps DS0's in a circuit switched network). We assume that that the time varying offered load at time $t$ is characterized by a nonstationary Poisson process with mean arrival rate $\lambda(t)$ at time $t$. This is consistent with the measurement results and models reported in [6], [11], [9].

The call holding (duration) time is assumed to be exponentially distributed, with mean, $1 / \mu$. Thus, the offered load, the arrival rate and the holding time are related by $a(t)=\lambda(t) / \mu$. Note that there is no restriction on the arrival pattern of traffic within a connection, only on the holding time of connections and the time between connection requests. A connection arrival, at time, $t$, finds capacity $C(t)$, if there is a free unit of capacity, in the present value of $C(t)$, to accommodate the connection, then the connection is accepted and uses a unit of bandwidth, otherwise, the connection is blocked and cleared. Note that $C(t)$ is time-dependent and represents the adjustable capacity in the network link. Under the assumptions above we have a time-dependent, nonstationary loss system which is a $\mathrm{M}(\mathrm{t}) / \mathrm{M}(\mathrm{t}) / \mathrm{C}(\mathrm{t}) / \mathrm{C}(\mathrm{t})$ type queue.

Obtaining closed form expressions for the general nonstationary behavior of queueing systems is extremely difficult, so we adopt a numerical solution approach using a fluid flow approximation. Specifically, we use the Pointwise Stationary Fluid Flow Approximation (PSFFA) method [19], [20]. Here, we derive the PSFFA model for the specific system under consideration. We define $x(t)$ as the state variable representing the average number of connections that are present on the link at time, $t$. Let $\dot{x}(t)=d x(t) / d t$ be the rate of change of the state variable with respect to time. From the flow conservation principle, the rate of change of the average number in the system is equal to the difference between the average arrival and departure rates. Let $f_{\text {in }}(t)$ and $f_{\text {out }}(t)$ denote the ensemble average number of connections in and out of the system at time $t$, respectively. Then the rate of change of the state variable can be related to the flow in and flow out by:

$$
\dot{x}(t)=-f_{\text {out }}(t)+f_{\text {in }}(t)
$$

This type of equation is referred to as a fluid flow equation [9], [19], [20]. To determine the particulars of the flow in and out of the system for the $\mathrm{M} / \mathrm{M} / \mathrm{C} / \mathrm{C}$ queue case, we let $\pi_{i}(t) ; i=0 ; 1, \ldots, C(t)$ denote the state probabilities of the system, with $\pi_{i}(t)$ representing the probability that there are $i$ connections in the system at time $t$. The flow into the system is just the offered load $\lambda(t)$ minus the portion of the offered load that is blocked. Hence we have:

$$
f_{\text {in }}(t)=\lambda(t)\left(1-\pi_{C(t)}(t)\right)
$$

where $\pi_{C(t)}(t)$, denotes the probability that a connection is blocked at time $t$, given the current capacity $C(t)$. The flow out of the queue $f_{\text {out }}(t)$, is the current utilization of the $C(t)$ servers and is given by

$$
f_{\text {out }}(t)=\mu \pi_{1}(t)+2 \mu \pi_{2}(t)+\ldots+C(t) \mu \pi_{C(t)}(t)
$$

Which can be shown to be equivalent to $f_{\text {out }}(t)=\mu x(t)$. Thus, the fluid flow model becomes:

$$
\dot{x}(t)=-\mu x(t)+\lambda(t)\left(1-\pi_{C(t)}(t)\right)
$$

Computing an exact solution for $\pi_{C(t)}(t)$ in the nonstationary case is difficult and we use the PSFFA approach of approximating it from the steady state formulas for the system under study [19], [20]. For the M/M/C/C system we use the steady state functional relationships to estimate $\pi_{C(t)}(t)$ as a function of $x(t)$. At steady state [8], the average number of connections in the system $x$, the offered load $a$ Erlangs and the blocking probability $\pi_{C}$ are related by:

$$
x=a\left[1-\pi_{C}\right]
$$

and

$$
\pi_{C}=E(a, C)=\frac{\frac{a^{C}}{C !}}{\sum_{k=0}^{C} \frac{a^{k}}{k !}}
$$

Note, that $\pi_{C}=E(a, C)$, where $E(a, C)$ is the well known Erlang-B loss formula. We assume the same functional relationships hold for the time varying behavior and we solve for $a(t)$ and $\pi_{C(t)}(t)$ using the equations above. Specifically, equation (5) is rewritten as:

$$
a(x(t))=\frac{x(t)}{1-E(a(x(t)), C(t))}
$$

Since equation (6) is now a function of $C(t)$, and the offered load, $a(t)$ is a function of $x(t)$, we get:

$$
\pi_{C(t)}(t)=E\left(a(x(t), C(t))=\frac{\frac{a(x(t))^{C(t)}}{C !}}{\sum_{k=0}^{C(t)} \frac{a(x(t))^{k}}{k !}}\right.
$$

Note that equation (7) is a fixed point equation which can be solved together with equation (8) in an iterative fashion to jointly determine $a(x(t))$ and $\pi_{C(t)}(t)$. Thus, the PSFFA model of the system is given by (4) together with (7) and (8).

The PSFFA model can be solved numerically to determine the time varying behavior of the system. We identify an initial condition for the state variable at time $t_{0}$ as $x\left(t_{0}\right)$ and an initial capacity value $C\left(t_{0}\right)$. The arrival rate is approximated by a constant over a small time step $\triangle t$, by $\lambda(t)=\lambda\left(t_{0}+\triangle t / 2\right)$ for $t \in\left[t_{0}, t_{0}+\triangle t\right]$. Then the blocking probability $\pi_{C(t)}(t)$ is approximated by a constant over $t \in\left[t_{0}, t_{0}+\Delta t\right]$ by solving (7) together with (8). The PSFFA model (4) can then be numerically integrated using a standard technique, such as, the Runge-Kutta-Fehlberg numerical method [2]. The numerical solution yields the value of the state variable at the end of the time interval, $x\left(t_{0}+\triangle t\right)$, which then becomes the initial condition for the next time step $\left[t_{0}+\Delta t, t_{0}+2 \triangle t\right]$. We then 
adjust the capacity and arrival rate for the new time step and the procedure is repeated for each interval in the time horizon. The numerical solution technique can be written in algorithmic form over $\left[t_{0}, t_{f}\right]$ as follows.

1) Initialization: set current time $t$, to $t=t_{0}$ establish the initial system occupancy $x(t)=x\left(t_{0}\right)$, system capacity $C(t)=C\left(t_{0}\right)$ and specify a time step $\Delta t$

2) Approximate the arrival rate $\lambda(t)$ by a constant $\lambda$ over $[t, t+\Delta t]$ with $\lambda=\lambda(t+\Delta t / 2)$

3) Approximate $\pi_{C(t)}(t)$ over $[t, t+\Delta t]$ by a constant $\pi_{C(t)}$ by solving (7) and (8) iteratively until the change in $a(x(t))$ does not exceed a prespecified $\epsilon$

4) Utilizing $x(t), \lambda$ (from step 2), $\pi_{C(t)}$ (from step 3 ), numerically solve the differential equation given by (5) over the small time interval $\Delta t$, and get the new system occupancy at time $t+\Delta t ; x(t+\Delta t)$.

5) Increment time, $t=t+\Delta t$. If $t<t_{f}$, go to 2 , else stop.

In order to evaluate the accuracy of the fluid flow model we compare it to exact results. For finite capacity Markovian queues the nonstationary behavior can be determined with high precision by integrating the associated Chapman Kolmogorov differential equation model [19]. Defining $p_{j}(t)$ as the probability of $j$ connections being in the system at time $t, \mu$ as the mean service rate, and $\lambda(t)$ as the time varying mean arrival rate, $C$ as the fixed system capacity, the Chapman-Kolmogorov differential equations [8] (CK model) are given by:

$$
\begin{aligned}
d p_{0}(t) / d t= & -\lambda(t) p_{0}(t)+\mu p_{1}(t) \\
d p_{j}(t) / d t= & \lambda(t) p_{j-1}(t)-(\lambda(t)+j \mu) p_{j}(t) \\
& \quad+(j+1) \mu p_{j+1}(t), \quad 0<j<C \\
d p_{C}(t) / d t= & \lambda(t) p_{C-1}(t)-C \mu p_{C}(t)
\end{aligned}
$$

This differential equation model can be solved numerically in a fashion similar to the solution of the PSFFA model. One approximates the arrival rate by a constant over a small time step and applies a standard numerical integration algorithm to solve the differential equations over the time step. This procedure is repeated over the time horizon as detailed in [19]. From the solution to the CK model for the time dependent state probabilities $p_{j}(t)$, one can directly determine the time varying mean number in the system using $x(t)=\sum_{i=0}^{N} i p_{i}(t)$ and the blocking probability using $p_{C}(t)$.

Following the literature ([6],[11], [13],[20]), we consider the nonstationary load to follow a sinusoidal pattern representing the cyclic load over a fixed time period (e.g., a day), specifically $\lambda(t)=A+B \sin (w(t+D))$ where $A, B, w$ and $D$ are constants. Figures 1 and 2 show typical nonstationary behavior of the system as determined by the PSFFA and CK models, in this case for the $M / M / 24 / 24$ queue (corresponding to a T1 line) with $\mu=1, x(0)=0$ and arrival rate $\lambda(t)=$ $15+3 \sin (0.1(t+20))$. From the figures one can see that the PSFFA models the system quite accurately.

\section{AdAPtive CAPACITy Allocation}

The proposed control scheme is based on the idea that the blocking probability should be maintained close to a

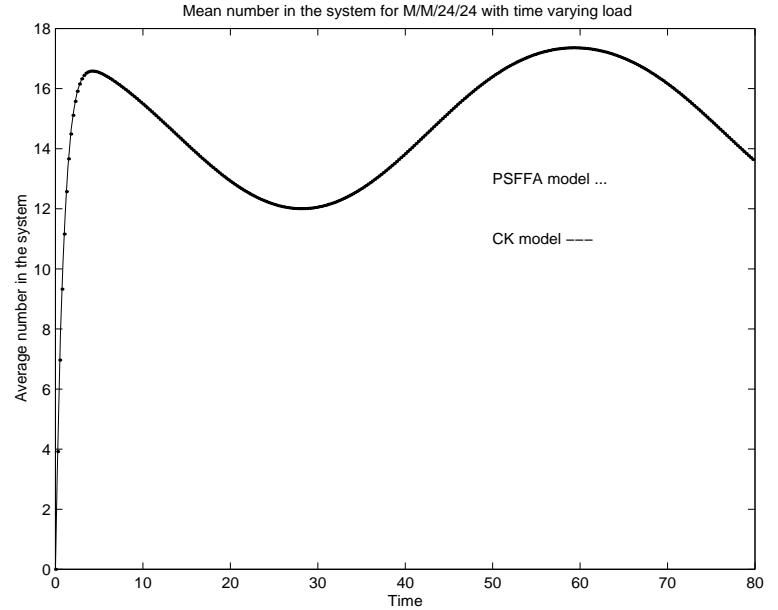

Fig. 1. Mean number in the system for nonstationary traffic.

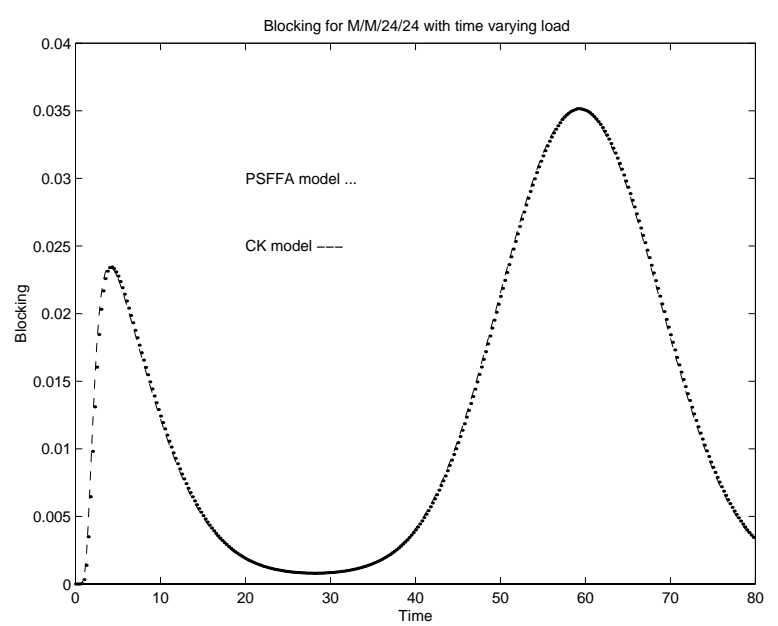

Fig. 2. Blocking Probability for nonstationary traffic.

desired target value or target trajectory. In other words, if the connection blocking rate is beyond an acceptable level, a request for additional capacity is desirable to provide an acceptable level of blocking. By the same token, if the current blocking is significantly lower than what is acceptable, it may indicate that this system may have too much idle bandwidth, thus it is desirable to release some of it. While heuristic capacity adjustment schemes along these lines have been proposed in the literature [7],[15]; they lack provable qualtities and their performance is sensitive to parameter settings.

Here we use the fluid flow model of the previous section together with results from Lyapunov stability theory to design a feedback based adaptive bandwidth controller that guarantees stability of the system around a desired blocking probability. Note, that the control is based on the fluid flow model rather than the CK model due to the scalability and simplicity of the fluid flow model. The stability based control approach adopted here, has recently been advocated in robotics and other nonlinear systems [12],[5]. The approach has the advantage 
of ensuring stability while allowing deviations around the desired target value in order to economize on the control action required. In the telecommunications context we note that service level agreements between customers and a transport network service provider typically provide guarantees on the call blocking rate over a time period (e.g., $2 \%$ call blocking during the peak busy hour). Thus it is not necessary to try and adjust the capacity to track the desired blocking probability exactly.

In designing the controller note that, since the blocking rate, $\pi_{C}(t)$, is related to the number of connections in the system, $x(t)$, as indicated in equation (5), we can control the blocking probability by controlling the number of connections. Hence, the desired target value of the blocking probability is translated to a corresponding target value for the number of connections. Using this parameter translation simplifies the computation and improves the ability to measure the feedback information as the number in system is easier to measure than the blocking probability. Next, we present the Lyapunov theorem for globally asymptotic stability [5] and then we show its application in determining the controller design:

Theorem 1: The equilibrium point of differential equation $\dot{e}(t)=f(t, e)$ is globally asymptotically stable if a Lyapunov function, $V(e)$, can be determined such that: (1) $V(e)$ is a continuously differentiable positive definite function, (2) $V(e)$ is negative definite and (3) $V(e) \rightarrow \infty$ as $\|e\| \rightarrow \infty$.

The proof of the stability theorem and its variations (i.e., stability in the sense of Lyapunov) are given in [5]. Here we use the stability theorem to craft a control that will guarantee the stability of the number of connections $x(t)$ in tracking a desired target number of connections $x_{d}(t)$. Specifically, let $\pi_{C}^{d}(t)$ denote the desired target blocking probability, which from (5) maps into $x_{d}(t)$ as the corresponding target number of connections in the system.

The error then can be defined as:

$$
e(t)=x_{d}(t)-x(t)
$$

Taking the derivative of both sides yields,

$$
\dot{e}(t)=\dot{x}_{d}(t)-\dot{x}(t)
$$

Substituting (4) in the above equation yields,

$$
\begin{gathered}
\dot{e}(t)=-\mu x_{d}(t)+\lambda(t)\left(1-\pi_{C}^{d}(t)\right)-\left(-\mu x(t)+\lambda(t)\left(1-\pi_{C}(t)\right)\right) \\
\dot{e}(t)=-\mu e(t)-\lambda(t)\left(\pi_{C}^{d}(t)-\pi_{C}(t)\right)
\end{gathered}
$$

Our control approach is to determine the capacity adjustment that ensures the error system defined by (12) is globally asymptotically stable as defined in Theorem 1. In determining our adaptive bandwidth controller we note that the parameters and variables are restricted as follows, $x(t) \geq 0, x_{d}(t) \geq 0$, $\lambda(t) \geq 0, \mu>0$ and $C$ is an integer. For the error system (12) to be stable, we must determine a Lyapunov function such that it meets the conditions of the stability theorem. Here we define a Lyapunov function by:

$$
V(e)=\frac{1}{2} e^{2}
$$

which is positive definite, as well as meeting the third condition of the stability theorem. The derivative of $V(e)$ is given by,

$$
\dot{V}(e)=e(t) \dot{e}(t)=e(t)\left(-\mu e(t)-\lambda(t)\left(\pi_{C}^{d}(t)-\pi_{C}(t)\right)\right)
$$

For stability, $V(e)$ must be negative definite function and we pick the contoller to ensure this. In particular we select $C(t)$ such that it results in

$$
\pi_{C}(t)=-\frac{k_{f} e(t)}{\lambda(t)}+\pi_{C}^{d}(t)
$$

where $k_{f}$ is a positive feedback controller gain. Substituting the controlled blocking rate $\pi_{C}(t)$ into (14) results in

$$
\begin{gathered}
\dot{V}(e)=-\mu e^{2}(t)-\lambda(t) e(t)\left(\pi_{C}^{d}(t)-\left(-\frac{k_{f} e(t)}{\lambda(t)}+\pi_{C}^{d}(t)\right)\right) \\
\dot{V}(e)=-\left(\mu+k_{f}\right) e^{2}(t)
\end{gathered}
$$

which is negative definite resulting in a stable system. Hence, the basic control is to calculate $\pi_{C}(t)$ from (15) and then determine the required $C$ from (6). Note that in determining the control, $0 \leq \pi_{C}(t) \leq 1$, which from (16) restricts the choice of the feedback gain $k_{f}$. In general, the smaller $k_{f}$, the closer the system will track the target probability, but at the expense of more capacity and frequent adjustments. Conversely, the larger $k_{f}$, the greater the variation allowed in the blocking probability and the greater the capacity gains.

The control formulation can be written in algorithmic form for operation over a time interval $\left(t_{0}, t_{f}\right)$ as follows:

\section{Adaptive Bandwidth Control Algorithm}

1) Initialization: Specify a control time step $\Delta t_{c}$, initial target blocking probability $\pi_{C}^{d}$, initial load $\lambda\left(t_{0}\right)$, mean call duration $\mu$, initial system occupancy $x(t)=x\left(t_{0}\right)$, and initial system capacity $C(t)=C\left(t_{0}\right)$. Determine the target number in system $x_{d}\left(t_{0}\right)$.

2) Estimate or measure the offered load $\lambda(t)$ and error $e(t)$ over $\left[t ; t+\triangle t_{c}\right]$. If the mean call duration $\mu$ is expected to vary, it can be measured as well.

3) Calculate $\pi_{C(t)}(t)$ from (15). Solve for the required capacity, $C(t)$, from (6), and update the link bandwidth. Update $x_{d}(t)$ from (5) for the next control time step.

4) Increment time, $t=t+\Delta t_{c}$. If $t<t_{f}$, go to 2 , else stop.

Note that there are two parameters in the control algorithm: the control time step $\triangle t_{c}$ which determines how frequently the capacity will be adjusted and $k_{f}$ which determines how closely the blocking rate should track the desired blocking value. The effects of varying these parameters is shown below. 


\section{PERformance AnAlysis}

In this section we present numerical results illustrating the performance of the proposed adaptive bandwidth scheme, additional results are given in [1]. The performance is estimated by incorporating the adaptive bandwidth control algorithm into the fluid-flow modeling framework presented in Section II. For all numerical solutions to differential equations, the fifth order Runge-Kutta routine provided in MATLAB was utilized. In the numerical solution various values for the time step $\Delta t$ (e.g., $\Delta t=0.1, \Delta t=0.01$, etc.) over which each integration is conducted were tried, until decreasing the time step resulted in no change in the results.

First we evaluated the performance of the proposed scheme in comparison to static bandwidth allocation. In our baseline experiments, the mean call holding time was $\mu=1$, the offered load followed a sinusoidal pattern $\lambda(t)=\widehat{\lambda}+(3$ $\sin (0.1(t+20)))$ with $\hat{\lambda}=15$, the desired target blocking probability was a fixed $\pi_{C}^{d}(t)=0.02 \forall t$, the control time step was $\Delta t_{C}=1$ and the feedback gain was set to $k_{f}=0.1$. The duration of each simulation was from $t_{0}=0$ to $t_{f}=120$. Note, that for the mean offered load of $\widehat{a}=\widehat{\lambda} / \mu=15$ Erlangs the fixed capacity allocation to achieve $\pi_{C}^{d}=0.02$ is $\mathrm{C}=23$. Similarly, at the peak load ( $a=18$ Erlangs), the capacity allocation to achieve $\pi_{C}^{d}=0.02$ is $C=26$. In Figure 3, we plot the probability of blocking versus time for the Lyapunov based adaptive capacity scheme along with the results of using a static capacity allocation at the peak rate $(\mathrm{C}=26)$ and the mean rate $(\mathrm{C}=23)$. As shown, the proposed Lyapunov scheme maintains the blocking rate roughly around 0.02 . Figure 4 shows the capacity allocation for the three schemes. Notice that the Lyapunov based scheme provides considerable capacity savings over peak rate allocation, while maintaining the blocking rate close to the desired value.

Secondly, the effect of the mean load on the performance of the Lyapnov based controller was investigated. In Figures 3 , and 5 we plot the probability of blocking versus time for the proposed adaptive capacity scheme with different average load values namely; $\widehat{a}=15$, and 100 . As shown in the figures, the blocking rate is maintained around 0.02 for each load with the variations being smaller for the larger load. It is important to observe, however, that the percentage of load deviation for each case is different. Specifically, when $\widehat{a}=15$ there is a deviation of $\pm 20 \%$ in the load, whereas, for $\widehat{a}=100$ there is a deviation of $\pm 3 \%$ in the load.

In the next set of experiments, we vary the desired target probability of blocking $\pi_{C}^{d}$ for the $\widehat{a}=15$ load case (i.e., $\lambda(t)=15+3 \sin (0.1(t+20)))$. Figure 6 , shows the probability of blocking results when $\pi_{C}^{d}=0.01$ and $\pi_{C}^{d}=0.03$. As shown in the figure, the blocking probability curves track around the specified target vales. Figure 7 shows the corresponding capacity adjustments. One can see the smaller the desired blocking rate, the larger the capacity required and the capacity value is changed more frequently. Notice, the controller performance is robust to changes in the target blocking rate, not requiring any parameter adjustment. Also, there is no requirement that

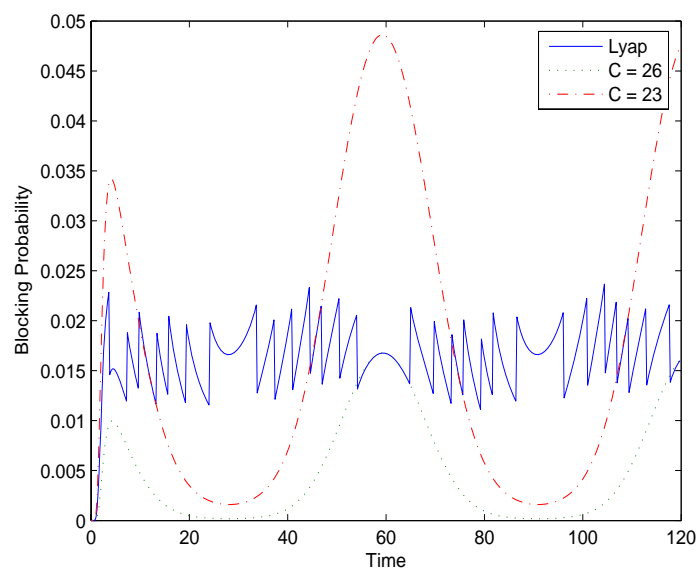

Fig. 3. Blocking Probability for average offered load $\widehat{a}=15$

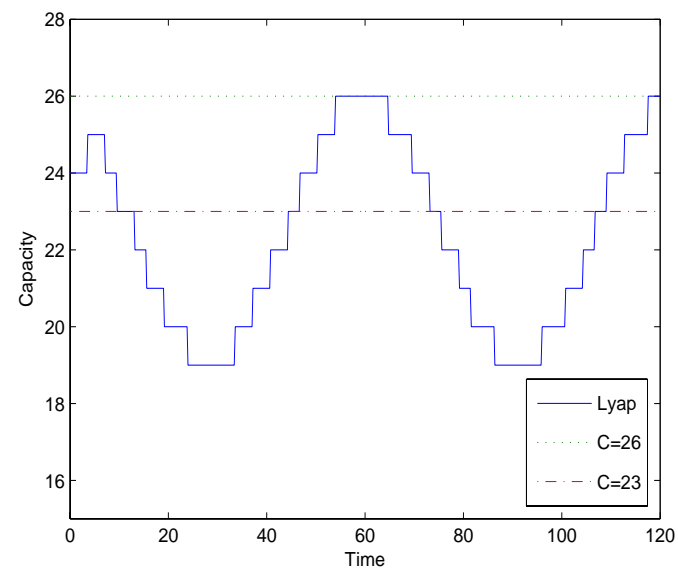

Fig. 4. Capacity allocation for average offered load $\widehat{a}=15$

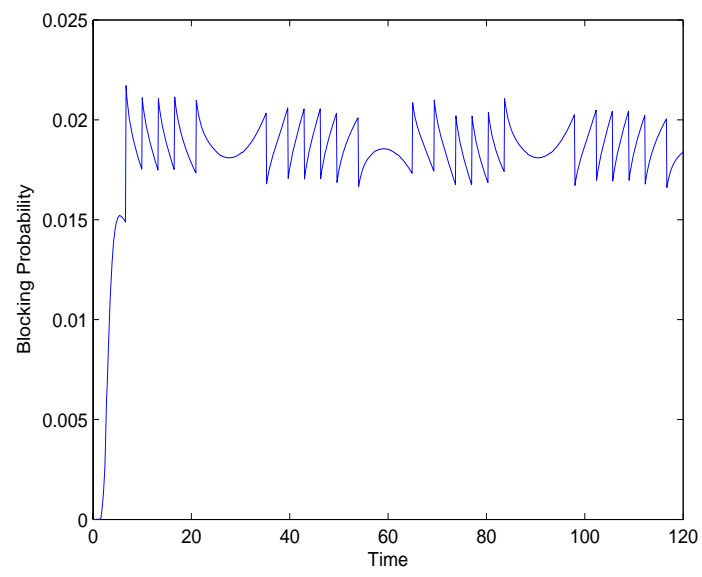

Fig. 5. Blocking Probability for the average offered load $\widehat{a}=100$

the target blocking rate be held constant. 


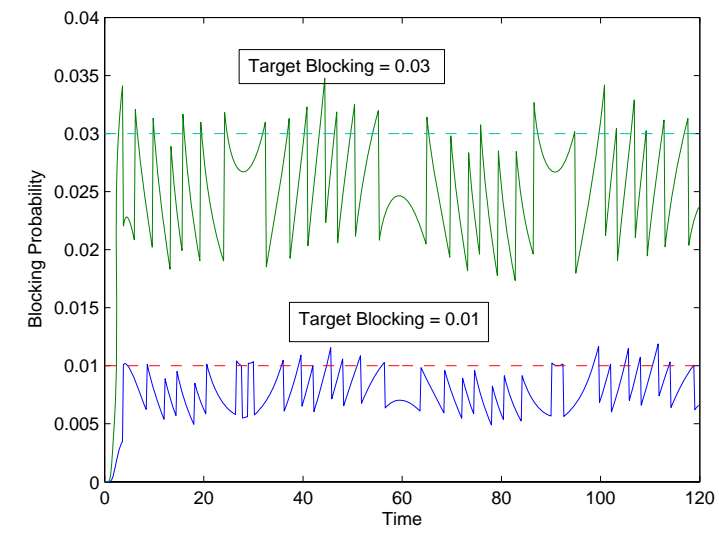

Fig. 6. Blocking behavior for two different target blocking rates

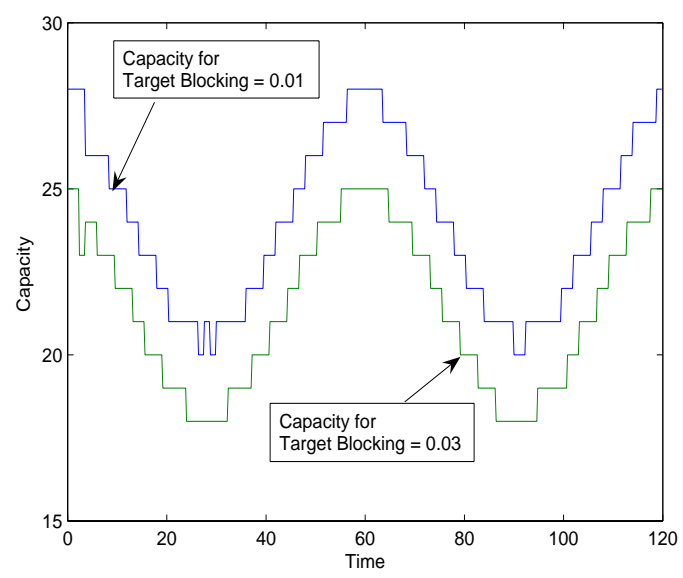

Fig. 7. Capacity allocation for two different target blocking rates

To evaluate the performance of the adaptive bandwidth scheme to the speed of variations in the offered load, we vary the frequency of the load, specifically, we set $a(t)=15+$ $3 \sin (\gamma(t+20))$ and $\gamma=0.05,0.1$ and 0.5 . The corresponding probability of blocking behavior is shown in Figures 8, 3 and 9. As shown in the figures, the blocking probabilities are maintained around the target blocking rate, with the high frequency case having the slightly worse performance. Notice that the time duration for the low freqency load simulation is longer than the other simulations.

The effect of varying the control time step $\Delta t_{c}$ was investigated for a variety of load scenarios. We illustrate the effect for the high frequency case with $\gamma=0.05$. The blocking behavior when the control time step is decreased to $\triangle t_{c}=0.2$ is shown in Figure 10. Notice that improvement in the blocking behavior in comparison to Figure 9. Clearly, the more frequent the changes in the systems load the more frequent the bandwidth control should be updated.

Lastly we compared our results with one of the adaptive bandwidth heuristics given in [7]. The heuristic scheme is

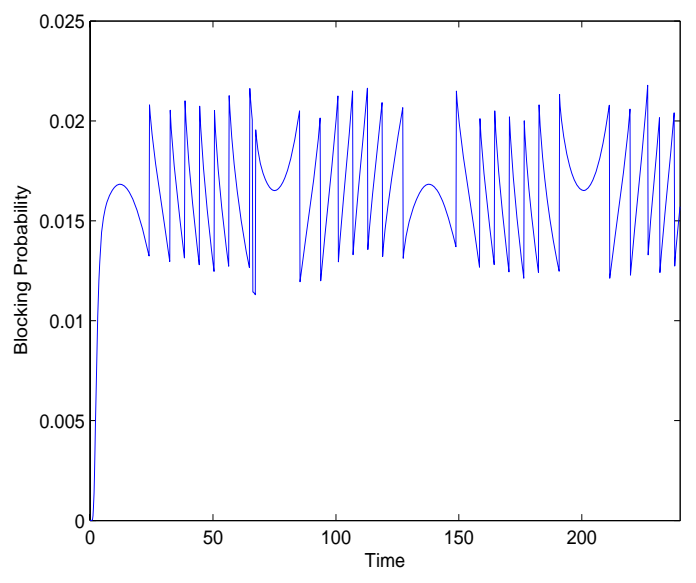

Fig. 8. Blocking behavior for the low frequency case $\gamma=0.5$

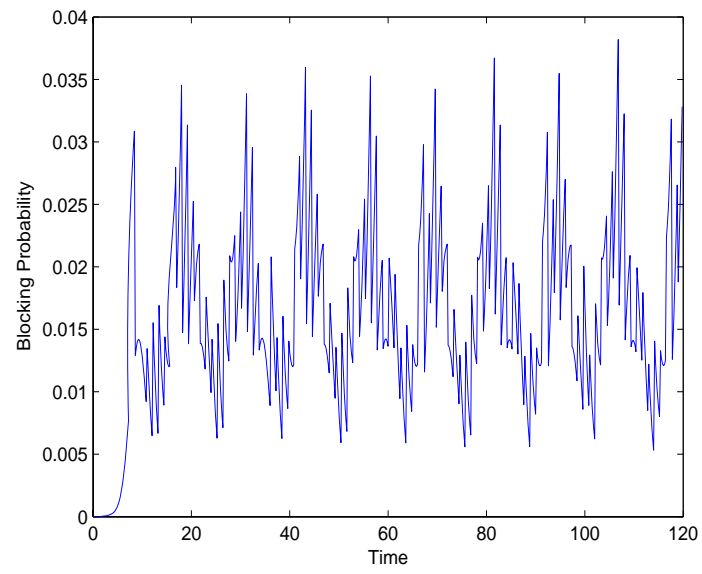

Fig. 9. Blocking behavior at high frequency load $\gamma=0.05$

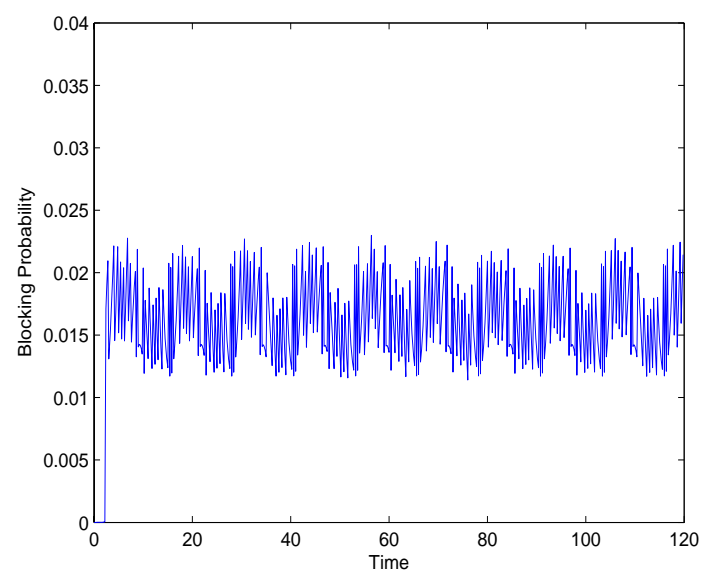

Fig. 10. Blocking behavior with $\gamma=0.05$ and $\Delta t_{c}=0.2$

built on the idea that if the connection blocking is beyond an 
acceptable level, or below a minimum threshold, a request for additional capacity, or release of it, respectively, is desirable to provide an acceptable level of blocking. In [7], the actual amount to be adjusted is pre-set to a value, $\mathrm{k}$, and the scheme can be summarized as follows. Let $b(t)$ be the blocking rate at time $t, b^{o b}$ denote the desired blocking rate and $b^{d}$ denote the allowable deviation from the desired blocking rate. The basic scheme is given as:

$$
\begin{aligned}
& \text { If }\left(b(t)<b^{o b}-b^{d}\right) \text { then } \\
& \quad C(t)=C(t)-k \\
& \text { Else if }\left(b(t)>b^{o b}+b^{d}\right) \text { then } \\
& \quad C(t)=C(t)+k \\
& \text { Else if }\left(b^{o b}-b^{d} \leq b(t) \leq b^{o b}+b^{d}\right) \text { then } \\
& \quad \text { No adjustment } \\
& \text { Endif }
\end{aligned}
$$

Note, that the scheme requires constant monitoring of the blocking probability which makes it difficult to implement as a large number of measurements are required to accurately estimate the blocking rate. Here for the purpose of comparison, we implemented the scheme above using the fluid flow model of Section II. In Figure 11 the blocking probability versus time for $\widehat{a}=15$ load case and $k=3$ is given. One can see that the heuristic results in large blocking rates. Comparing Figure (11) with the results of our proposed scheme for the same load in Figure 4, one can see the that Lyapunov scheme outperforms the one in [7]. Furthermore, the Lyapuov based scheme does not require extensive parameter tuning and has the advantage that the the capacity is automatically adjusted to meet the stability criteria.

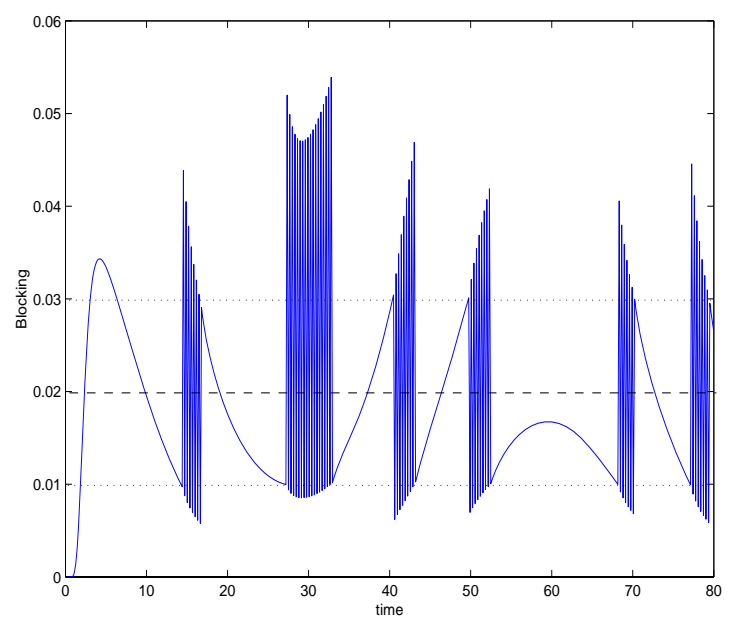

Fig. 11. The blocking behavior of [7] scheme with $\mathrm{k}=3$, and offered load of $\lambda(t)=15+3 \sin (0.1(t+20))$

\section{Conclusions}

In this paper, we developed a novel call leve adaptive capacity allocation scheme for a network that operates in a loss mode. The capacity allocation scheme was determined by the application of Lyapunov Stability Theory to a fluid flow model of link queuing behavior. The resulting adaptive capacity control scheme maintains the connection blocking rate around a desired target blocking value so that the resulting error system is globally asymptotically stable. Numerical results showing the effectiveness of the proposed scheme and its robustness to load variations were given. The extension of the control approach to the multiple heterogeneous traffic classes scenario is given in [1].

\section{REFERENCES}

[1] H. Bin-Abbas,"Adaptive Capacity Allocation in MPLS networks", Ph.D. dissertation, University of Pittsburgh, Dec., 2004.

[2] R. Burden, J.D. Faires, and A. C. Reynolds, Numerical Analysis, Second Edition. Prindle,Weber \& Schmidt, Boston, MA, 1981.

[3] D. Cavendish, M. Gerla, and S. Mascolo, "A Control Theoretical Approach to Congestion Control in Packet Networks," IEEE/ACM Trans. on Networking, Vol. 12, No. 5, pp. 893-906, Oct., 2004.

[4] D. Cavendish, K. Murakami, S. Yun, O. Matsuda, M. Nishihara, "New Transport Services for next generation SONET/SDH systems," IEEE Communications Magazine, Vol. 40, No. 5, pp. 80-87, May, 2002.

[5] W. E. Dixon, A. Behal, D. M. Dawson, and S. Nagarkatti, "Nonlinear Control of Engineering Systems: A Lyapunov-Based Approach", Cambridge, England, Birkhäuser, 2003.

[6] S. Eick, W. Massey, and W. Whitt, "Nonstationarity in Offered Traffic to the AT \& T Long Distance Network," AT \& T Symposium on Performance Analysis and Teltraffic Restoration: Theory and Application. Homedel, N.J., June 1990.

[7] B. Groskinsky, D. Medhi and D. Tipper,"An Investigation Of Adaptive Capacity Control Schemes In A Dynamic Traffic Environment", IEICE Tran. on Communications, Vol. E84-B, pp. 263-274, February 2001.

[8] D. Gross and C. Harris, Fundamentals of Queueing Theory, Third Edition, John Wiley, \& Sons New York, NY 1998.

[9] X. Gu, K. Sohraby, and D. Vaman, Control and Performance in Packet, Circuit, and ATM Networks, Kluwer Academic Publishers, Norwell, MA, 1995.

[10] C. Hollot, V. Mishra, D. Towsley, and W. Gong,"Analysis and Design of Controllers for AQM Routers Supporting TCP Flows," IEEE Trans. on Automatic Control, Vol., 47., No. 6., June 2002

[11] D. Jagerman, "Nonstationary Blocking in Telephone Traffic," Bell System Technical Journal, Vol. 54, pp.625-661, 1975.

[12] F. Lewis, D. Dawson, C. Abdallah, Robot Manipulation Control: Theory and Practice, Marcel Dekker, New York, 2004.

[13] W. Massey, "The Analysis of Queues with Time-Varying Rates for Telecommunication Models", Telecommunication Systems, Vol. 21, pp. 173-204, 2002.

[14] A. Pitsillides, P. Ioannou, M. Lestas, L. Rossides, "Adaptive Nonlinear Congestion Controller for a Differentiated Services Framework," ACM/IEEE Transactions on Networking, Vol. 13., No. 1, pp.94-107, Feb., 2005.

[15] S. Shioda, H. Saito, and H. Yokoi, "Sizing and Provisioning for Physical and Virtual Path Networks Using Self-Sizing Capability," IEICE Transactions on Communications, Vol. E80-B, No. 2, pp. 252-262, 1997.

[16] P. Siripongwutikorn, S. Banerjee, and D. Tipper. A Survey of Adaptive Bandwidth Control Algorithms, IEEE Communications Surveys \& Tutorials, Vol. 5, No. 1, pp. 2-14, Third Quarter, 2003.

[17] P. Siripongwutikorn, S. Banerjee and D. Tipper, "Fuzzy Based Adaptive Bandwidth Control for Loss Guarantees," (Special Issue on Adaptive Learning Systems in Communication Networks) IEEE Transactions on Neural Networks, Vol. 16, No. 5 , pp.1147-1162, Sept., 2005

[18] K. Mase and M. Imase, "An Adaptive Capacity Allocation Scheme in Telephone Networks," IEEE Transactions on Communications, Vol. Com-30, No.2, pp. 354-359, Feb., 1982.

[19] D. Tipper, M.K. Sundareshan, M.K. "Numerical Methods for Modeling Computer Networks Under Nonstationary Conditions," IEEE Journal on Selected Areas in Communications, Vol. 8, No. 9, pp.1682-1695, 1990.

[20] W. Wang, D. Tipper, and S. Banerjee, "A Simple Approximation for Modeling Nonstationary Queues," Proceedings IEEE INFOCOM'96, pp. 2d.3.1-2d.3.8, 1996 